\title{
Role of Huntington's Disease Protein in Post-Transcriptional Gene Regulatory Pathways
}

\author{
Brady P. Culver and Naoko Tanese \\ NYU School of Medicine \\ USA
}

\section{Introduction}

This chapter will focus on the potential role that misregulation of post-transcriptional control of gene expression could have on the development or progression of Huntington's disease (HD). Every cell in our bodies possesses the same genetic material, and yet every cell is not the same. We also all know that the tremendous diversity of biology present within each individual is accomplished through unique patterns of gene expression on a cell-by-cell basis. Of course the timing and amounts of gene expression also contribute to this diversity of phenotype and function. The complexity, however, goes even deeper. Within individual genes there is information to produce multiple different messenger RNAs and often multiple different proteins, each with different functional implications. We see then, that the multi-step process of gene expression using a number of genes only modestly greater than what is found in certain species of ciliates is capable of generating a being of vastly more complicated biology. With this complexity in mind, it is therefore possible that small defects at any of the steps of gene expression could have deleterious consequences on the identity, ability to appropriately respond to environmental cues, and on the survival of cells.

In HD, certain parts of the brain are primarily affected over others. Furthermore, the Huntington's disease gene product huntingtin $(\mathrm{Htt})$ is ubiquitously expressed. So how is specificity of the disease manifested when the mutant protein is present everywhere in our bodies at all times? One possible explanation for this observation is that the expression of specific genes important for the survival and function of the cells affected in HD are disrupted to a more significant extent than others. These alterations need not be drastic; rather they are more likely to be the result of an accumulation of small changes, which over time could lead to the dysfunction or death of particular types of neurons. There is already a large body of research on the role of $\mathrm{Htt}$ in transcriptional control, and many studies have identified reproducible alterations in gene expression patterns between mouse models of HD and postmortem human HD patient brain samples (Hodges et al. 2006; Seredenina and Luthi-Carter 2011). While we do not discount this potential mechanism, we suggest that mutant Htt may also influence gene expression at steps downstream of transcription.

This hypothesis, although unique amongst HD researchers, is either gaining traction or is already widely accepted as the basis for other neurodegenerative diseases. These diseases would affect the processing of multiple messenger RNAs and present with a broad 
phenotypic spectrum, reflecting the loss of function or aberrant processing of specific mRNAs. For example, amyotrophic lateral sclerosis (ALS) results from the death of cortical motor neurons and the spinal cord motor neurons on which they synapse. Although the majority of ALS cases have no genetic predisposition, a small percentage of cases have been linked to mutations in the RNA binding proteins TDP-43 and TLS (Lagier-Tourenne, Polymenidou, and Cleveland 2010). However, in both genetic (TDP-43 driven) and sporadic cases of ALS, TDP-43 forms abnormal intracellular aggregates, which are thought to influence the expression patterns of mRNAs dependent upon TDP-43 function for their normal post-transcriptional processing (Mackenzie et al. 2007). Similarly, Fragile X syndrome (FXS) and the related Fragile X tremor ataxia syndrome (FXTAS) are also known to result from mutations in the Fragile $X$ gene (FMR1) whose protein product is involved in translational control of mRNAs to which the protein is bound (Willemsen, Levenga, and Oostra 2011). These examples and others argue that the dysfunction of RNA binding proteins can produce cell-type specific effects based on the RNA binding proteins affected and their associated RNAs. Here we provide an overview of the steps at which gene expression may be controlled downstream of transcription, examples in which each of these processes may be perturbed in other neurodegenerative diseases, and review the evidence implicating $\mathrm{Htt}$ in control of post-transcriptional gene expression. We aim to incite enthusiasm in the reader for this underappreciated hypothesis and cite the numerous parallels between HD and other neurodegenerative diseases involving the dysfunction of normal post-transcriptional RNA processing.

\section{Gene expression is controlled at multiple steps downstream of transcription}

Transcription produces a full-length RNA copy of the DNA sequence of a gene, which is heavily edited through removal of intronic sequences, the joining of exonic sequences, the cleavage of the mRNA at specific sites, and the addition of elements not coded for in the genome. The processed RNA must then be exported from the nucleus to be translated. Upon nuclear export, mRNAs may be stored in a translationally repressed state bound by RNA binding proteins until this translational repression is relieved, or they may be immediately translated. Translationally repressed mRNAs can be trafficked to distant sites within the cell to impart an additional level of control to gene expression. mRNAs have a finite lifespan and the levels of mRNA can be controlled through degradation in addition to rates of transcription. Each of these steps is controlled by the activity of specific proteins, which ultimately enable a tight control of protein content, amounts, and location within the cell.

\subsection{Alternative patterns of RNA splicing produce multiple messages from a common gene}

Alternative splicing is the process by which different exons are joined together to form different sequences from the same precursor mRNA (pre-mRNA). The different patterns of exon joining in alternatively spliced transcripts are determined by sequences contained within the introns of pre-mRNAs and the presence or absence of the proteins that recognize these sequences, although sometimes exons themselves also play a role in this process (Wahl, Will, and Luhrmann 2009). Regardless of the location of the cis-acting elements within pre-mRNAs, specific RNA binding proteins promote the inclusion or exclusion of 
particular exons. These exons may contain protein-coding sequence or untranslated regions (UTRs) if they are present outside of the main open reading frame (ORF). Failure to remove an intron or the exclusion of a particular exon(s) can produce mRNAs that are targeted for degradation before any protein can be produced from these messages (Rebbapragada and Lykke-Andersen 2009). Most human genes are composed of multiple exons (Venter et al. 2001); therefore, the splicing process must be carefully orchestrated to ensure the generation of a meaningful and high fidelity transcript. Furthermore, most human genes are alternatively spliced, which vastly increases the diversity of RNAs and proteins coded for in the entire genome.

\subsubsection{Alternative splicing generates different protein isoforms}

Splicing events are catalyzed by a large ribonucleoprotein complex called the spliceosome (Wahl, Will, and Luhrmann 2009). Specificity is imparted through the action of distinct splicing factors that recognize particular sequence elements within a pre-mRNA and recruit the spliceosome to these sites. Additionally, splicing factors may mask individual splice sites so they are not included, or spliced out of the resultant mRNA. The activity of these splicing factors is required for the process of alternative splicing as well as to ensure the generation of an intron-free mature mRNA. Alternative splicing can change the amino acid coding potential of an mRNA and thereby generate multiple different proteins from a single gene. Proteins are often composed of modular domains. For example, a protein may harbor a membrane targeting sequence at its $\mathrm{N}$-terminus and a catalytic domain at its $\mathrm{C}$-terminus. Therefore, an mRNA encompassing both of these features would produce a membrane bound protein with catalytic activity. If the membrane-targeting domain of a hypothetical protein were contained within a single exon, then omission of this exon in an alternatively spliced version of this mRNA would produce a cytoplasmically localized protein with catalytic activity (Figure 1).

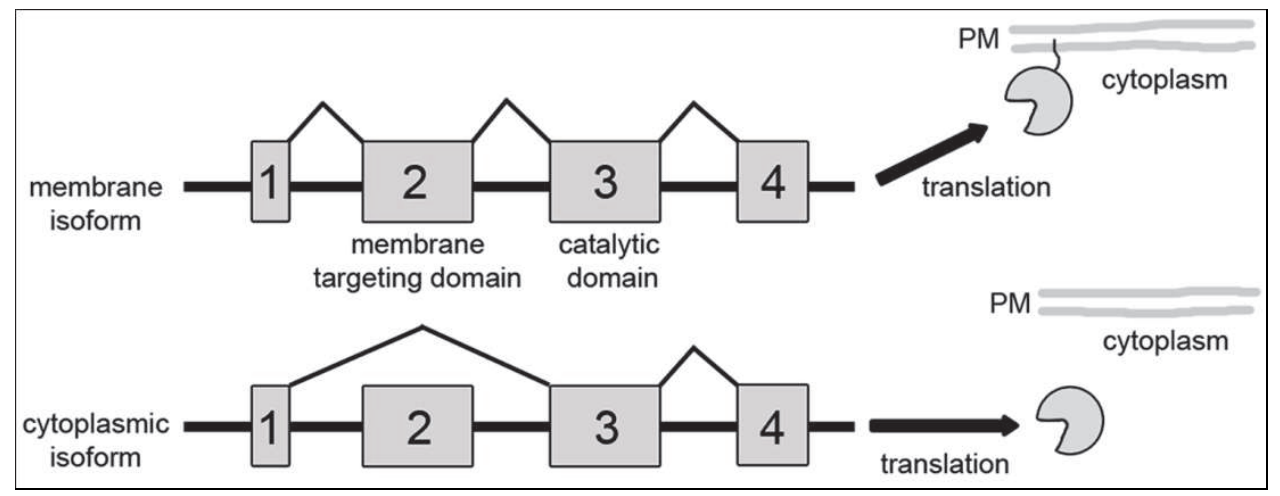

Fig. 1. Alternative splicing can generate different protein isoforms from a single gene. The cartoon illustrates a hypothetical gene with 4 exons (grey boxes numbered 1-4). The solid line represents the unspliced transcript, with the lines above indicating the splicing pattern of the hypothetical mRNA. Inclusion of exon 2 results in the translation of a protein with a plasma membrane (grey squiggly lines, PM) -targeting domain. Exclusion of exon 2 produces a cytoplasmically localized protein. 
The protein tau, which is one of the main components of the neurofibrillary tangles in dementia and Alzheimer's disease, is present in as many as 30 different isoforms within neurons, all of which are generated through alternative splicing (Andreadis 2011). Tau helps organize axonal microtubules and functions as a cytoskeletal scaffold in post-synaptic densities (Pritchard et al. 2011). Alterations in the ratio of tau isoforms are the cause of familial cases of frontotemporal lobar degeneration (FTLD) (Gasparini, Terni, and Spillantini 2007). These mutations do not change the coding potential of the tau gene, but instead alter the frequency of alternative splicing events such that appropriate stoichiometry of tau isoforms is disrupted.

\subsubsection{Defects in mRNA splicing lead to abnormal protein translation or mRNA degradation}

The inclusion of an intron(s) within a mature mRNA can lead to its degradation or the translation of a protein with an unintended amino acid sequence. Splicing aberrations are normally detected by the nonsense-mediated decay (NMD) pathway. This pathway recognizes mRNAs that contain a premature termination codon and targets them for destruction. Premature termination codons are recognized by the context in which they are found, vis-à-vis the presence of protein complexes on the transcript at defined positions. Normally, mature mRNAs are marked at splice sites by proteins of the exon-exon junction complex (EJC) (Rebbapragada and Lykke-Andersen 2009). If the NMD protein machinery encounters a stop codon upstream of an EJC, then this signals that the termination codon may be premature. However, the positioning of other factors on the mRNA may prevent NMD from occurring. In short, mRNAs are bound by proteins that recognize specific elements within their sequence. The positioning of these elements provides a context in which to determine whether the message has been appropriately processed. mRNAs that escape the NMD pathway are available for translation and when translated can provoke unforeseen cellular responses. Figure 2 illustrates the consequences of errors in splicing.

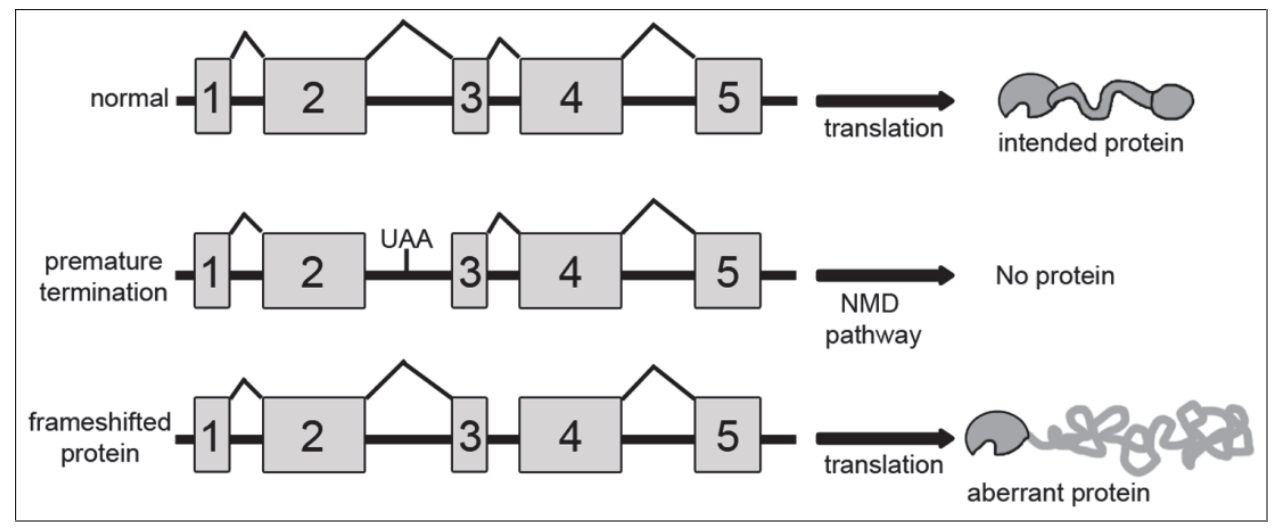

Fig. 2. Errors in splicing can lead to mRNA degradation or aberrant protein synthesis. The cartoon shows how errors in splicing can lead to the inclusion of an intron, which may contain a termination codon (UAA) and activate the NMD pathway. Alternatively, the intron may not contain a premature termination codon, but produce a frame-shifted transcript coding for an abnormal protein. 
In ALS, aberrant splicing results in the inclusion of introns in the mature mRNA for the intermediate filament protein peripherin (Xiao et al. 2008). Peripherin is present within the protein aggregations observed in ALS patient tissue and overexpression of peripherin is sufficient to induce neurodegeneration in transgenic mice (Robertson et al. 2003). Furthermore, expression of the aberrant transcript in cultured motor neurons resulted in its aggregation, and was associated with the death of these cells (Robertson et al. 2003). These findings suggest that the abnormal splicing events can trigger neurodegeneration in a cell type specific manner.

\subsection{Non-protein coding sequences influence stability, location, and translational potential of mRNAs}

Eukaryotic mRNAs contain untranslated regions (UTRs) at their $5^{\prime}$ and $3^{\prime}$ ends that contain information used in determining the stability, translational potential, and location of an RNA. As the name suggests, untranslated regions of an mRNA are not translated into protein, but serve as cis-acting elements within an mRNA that provide binding surfaces for other molecules through the secondary structural elements conferred by the sequence of the UTR (Spriggs, Bushell, and Willis 2010). While exons most often contain the information required for the assembly of the encoded protein, they also include UTRs at the $5^{\prime}$ and $3^{\prime}$ end of a mature mRNA. Sometimes different $5^{\prime}$ and $3^{\prime}$ UTRs for the same mRNA arise from alternative splicing events, as has been observed for the brain derived neurotrophic factor, BDNF (Pruunsild et al. 2007). Alternative versions of 3' UTRs may also be generated through the cleavage of precursor mRNAs in anticipation of poly-A (poly-adenosine monophosphate) tail addition (Hughes 2006). The vast majority of mRNAs include a stretch of poly-A residues at their $3^{\prime}$ end that are not coded for in the genome and are added posttranscriptionally. The poly-A tail protects the mRNA from degradation at the $3^{\prime}$ end and is involved in initiation of protein translation through its interaction with poly-A binding protein (PABP) (Lemay et al. 2010). Many pre-mRNAs may be poly-adenylated at multiple positions based on the presence of multiple poly-A signal sequences in the pre-mRNA (Tian et al. 2005). The selection of the cleavage site on the pre-mRNA can therefore directly determine the extent and content of the $3^{\prime}$ UTR contained within an mRNA. We provide a brief overview of the functions of $5^{\prime}$ and $3^{\prime}$ UTRs and highlight examples where perturbations in these processes may contribute to neurodegeneration.

\subsubsection{The 5' UTR functions in mRNA translation}

5' UTRs typically contain sequences necessary for the initiation of translation. The $5^{\prime}$ end of most mammalian mRNAs is capped by a modified ribonucleotide, the m7G cap. This modified nucleotide is attached to the $5^{\prime}$ end of the mRNA through an atypical $5^{\prime}$ to $5^{\prime}$ linkage. The $\mathrm{m} 7 \mathrm{G}$ cap is bound by a translation initiation factor complex (eIF4E), which is then used to circularize the transcript through binding to PABP. Circularization is thought to improve the efficiency of translation (Gingras, Raught, and Sonenberg 1999). In addition to the $5^{\prime}$ cap, $5^{\prime}$ UTRs often contain upstream open reading frames (uORFs). The presence of a $\mathrm{uORF}$ is usually inhibitory; a ribosome scanning along the mRNA will encounter the start codon of a $\mathrm{uORF}$ and begin translation at this position. This results in fewer ribosomes recognizing the main ORF start codon and decreased protein translation. uORF-encoded polypeptides can also directly inhibit translation by binding to the ribosome and preventing the translation of the major downstream ORF (Lovett and Rogers 1996). 
Beta site APP-cleaving enzyme 1 (BACE1) cleaves the amyloid precursor protein (APP) producing the toxic fragment that forms the amyloid plaques that define Alzheimer's disease (Sisodia 1992). Elevated levels of BACE1 protein expression are normally kept in check by the presence of six uORFs (Zhou and Song 2006). Alzheimer's disease patients typically display elevated levels of BACE1 protein compared with unaffected individuals, but the levels of BACE1 mRNA do not always reflect this (Mihailovich et al. 2007). This suggests that translational efficiency of BACE1 may be enhanced in Alzheimer's disease compared with unaffected cases. These uORFs are therefore thought to be important in keeping the levels of BACE1 protein low.

5' UTRs also typically contain highly structured regions, which can be bound by proteins that recognize these structures. Protein binding to the secondary structures within the $5^{\prime}$ UTR can promote 5' m7G cap-independent translation initiation (Pickering and Willis 2005). In this context, these secondary structures are known as IRES (internal ribosome entry sites). These sites are used under conditions of cellular stress when the translation of many other proteins is globally inhibited (Spriggs, Bushell, and Willis 2010). Global translational inhibition is mediated through the phosphorylation of eIF2A. eIF2A can be phosphorylated by four different kinases (PKR, PERK, GCN2, and HRI), which are activated by different cellular stresses. Phosphorylated eIF2A inhibits translation by preventing recycling of initiation complexes to translation start sites. The presence of an IRES in an mRNA can bypass this translational repression by recruiting ribosomes to start codons by an eIF4Aand m7G cap-independent mechanism (Spriggs, Bushell, and Willis 2010).

Fragile $\mathrm{X}$ syndrome (FXS) and fragile $\mathrm{X}$ tremor ataxia syndrome (FXTAS) are caused by differing degrees of a CGG repeat expansion at the $5^{\prime}$ non-protein-coding end of the fragile $X$ mental retardation 1 (FMR1) gene. The extent of CGG repeat expansion determines the disease; longer CGG expansions (> 200 repeats) result in FXS and shorter CGG expansions (55-200) result in FXTAS. The repeat expansion lengths of FXS silence transcription of FMR1, effectively producing a null mutation through increased methylation of the FMR1 gene promoter. By contrast, the shorter CGG expansions of FXTAS do not result in transcriptional silencing, but instead produce an mRNA containing a large stem-loop structure composed of CGG repeats in the 5' UTR (Willemsen, Levenga, and Oostra 2011). Transgenic mice expressing FXTAS-correlated CGG repeat lengths, outside of a protein translation context, phenocopy symptoms of the human condition, which indicates that the RNA itself may be the toxic agent in FXTAS (Van Dam et al. 2005). The stem-loop generated by expanded CGG repeats in the $5^{\prime}$ UTR of FMR1 could act as a sponge to pull the RNA binding proteins away from their normal targets and therefore affect the processing of other RNAs normally bound by these RNA binding proteins (Jin et al. 2003). This mechanism implies that the processing of many other RNAs may be affected by the single mutation in FMR1.

\subsubsection{The 3' UTR functions in mRNA stability and defines its localization}

3' UTRs are typically much longer than 5' UTRs and therefore contain significantly more information that can be used to affect the fate of mRNA to which they are attached. This information is decoded from the 3' UTR sequence by the binding of proteins or other RNAs to the $3^{\prime}$ UTR. A combination of sequence and secondary structure allows specific proteins to bind to 3' UTRs, where they affect the stability, localization, and translational potential of the mRNA. All of these processes are important regulators of gene expression, and defects 
in any one of these processes may lead to cell dysfunction and undesirable effects depending on the affected mRNAs (Andreassi and Riccio 2009).

Recently, the role of non-coding RNAs (ncRNAs) in the regulation of gene expression has received considerable attention. ncRNAs contribute to the regulation of gene expression at both the transcriptional and post-transcriptional steps. As nucleic acids, ncRNAs are able to form base-pair interactions with perfect or imperfect complementarity. This feature provides a simple mechanism to allow for the targeting of specific sequences by these ncRNAs. ncRNAs could therefore serve as an adaptor molecule to facilitate RNA-protein interactions (Mattick and Makunin 2006). In this model, a protein would have an affinity for either a sequence or a structural feature present within the ncRNA. The ncRNA in turn would have sequence elements within it that allow it to recognize and form base-pair interactions with distinct RNA(s). In this manner, an ncRNA could allow a protein to interact with a large variety of messages. No other system is as well characterized with regards to this phenomenon as the RNA interference pathway. In this pathway, small RNAs of 21-23 nucleotides in length recognize sequences in a target mRNA, and through base-paired interactions direct the assembly of a protein complex called RISC (RNA-induced silencing complex) onto the matched mRNA. Perfect complementarity between the small RNA (siRNA) and RNA target leads to cleavage and degradation of the RNA mediated by the endonucleolytic activity of the protein Argonaute 2 (Ago2) (Siomi and Siomi 2009). RNAi (RNA interference) has now become an invaluable tool in experimental molecular biology and has exciting potential therapeutic applications. Most small RNAs or microRNAs (miRNAs) present in mammalian cells, however, have imperfect sequence complementarity with their targets resulting in the translational silencing of the affected message through Ago family members Ago1, 2, 3, and 4 (Siomi and Siomi 2009), or degradation of target mRNAs . Current estimates suggest that around $60 \%$ of all human genes are regulated by miRNAs (Friedman et al. 2009). miRNAs and components of the RISC complex are found in dendrites where they repress the translation of synaptic proteins (Swanger and Bassell 2011). Although the effects of miRNAs on protein levels are subtle, the elimination of miRNAs from adult brain results in a neurodegenerative phenotype in mice (Hebert et al. 2010).

Changes in RNA stability may contribute to the pathology of ALS. The brains of ALS affected individuals possess intracellular accumulations of neurofilament proteins. Neurofilament proteins come in three different isotypes: neurofilament heavy, medium, and light, all encoded by separate genes. The stoichiometry of neurofilaments is hypothesized to be important to prevent their aggregation ( $\mathrm{Xu}$ et al. 1993). TDP-43 stabilizes the neurofilament light chain (NFL) mRNA by binding to its 3'UTR (Strong et al. 2007). As discussed in the introduction, TDP-43 is present within intracellular inclusions in ALS. This sequestration of TDP-43 may abrogate its binding to the NFL mRNA and thus decrease the stability of the mRNA. Indeed, NFL mRNA levels are reduced in ALS patient brains (Volkening et al. 2009). Therefore, this reduction in mRNA could translate to a reduction in NFL protein, altered neurofilament stoichiometry, and protein aggregation.

Myotonic dystrophy type 1 (DM1) is caused by a CTG repeat expansion in the 3' UTR of the dystrophia myotonica-protein kinase (DMPK) gene (Mahadevan et al. 1992). This repeat expansion results in the sequestration and aggregation of CUG-expanded RNA in the nucleus. Here the protein muscleblind-like (MBNL) binds to the CUG-expanded RNA and is prevented from performing its normal role in splicing (Jiang et al. 2004), which is similar to 
the proposed mechanistic explanation for FXTAS. Additionally, expression of a CUG repeatexpanded RNA was sufficient to cause the formation of large RNA and protein aggregations called stress granules in cell culture (Huichalaf et al. 2010). Stress granules are sites of RNA storage, where translation is inhibited and RNAs are kept from a potentially damaging cytoplasmic environment (Buchan and Parker 2009). Stress granules contain a heterogeneous population of mRNAs, and therefore the induction of the stress granule assembly in response to CUG-expanded RNA expression could affect the translation of many different mRNAs. Could CAG-expanded RNA expression in Huntington's disease produce a similar response?

\subsubsection{The poly-A tail contributes to stability and translational potential of an mRNA}

Polyadenylation is a requisite step in the biogenesis of most mRNAs. This modification serves to protect mRNAs from degradation by $3^{\prime}$ to $5^{\prime}$ exonucleases (Mangus, Evans, and Jacobson 2003). This protection is imparted to the mRNA through the binding of PABP to the poly-A sequence. As a polyadenylated mature mRNA ages, the length of the poly-A tail shrinks. This poly-A shortening is analogous to the process that occurs at telomeres as a cell ages. Just as a critically short telomere signals senescence, a critically short poly-A tail is unable to stave off the exonucleases wishing to make a lunch of it, and it is degraded (Meyer, Temme, and Wahle 2004). The poly-A tail therefore helps to establish a lifespan for RNAs. Polyadenylation most often occurs in the nucleus, but can also take place in the cytoplasm. Cytoplasmic mRNAs lacking a poly-A tail or possessing a shortened poly-A tail are sequestered in ribonucleoprotein particles until they are acted upon by a cytoplasmic polyadenylation element binding protein (CPEB), which promotes poly-A tail extension and subsequent translation (Richter 2007). The addition and function of the poly-A tail therefore provide yet another step at which gene expression may be regulated.

Brain derived neurotrophic factor (BDNF) possesses two cytoplasmic polyadenylation elements in its 3' UTR generating short and long forms of mRNAs. One of these elements is required for constitutive BDNF mRNA trafficking to dendrites, while the other is important for activity dependent trafficking (Oe and Yoneda 2010), although different conclusions were drawn from another study (An et al. 2008). BDNF plays important roles in the health and survival of neurons, and the protein is present at reduced levels in both Alzheimer's disease and HD patient brain tissue (Ferrer et al. 2000; Narisawa-Saito et al. 1996). In Alzheimer's disease, BDNF mRNA levels are reduced, and the severity of BDNF mRNA reduction correlates with the $A \beta$ aggregation size in mouse models of AD (Peng et al. 2009).

The autosomal dominant disorder, oculopharyngeal muscular dystrophy (OPMD) is caused by a GCG repeat expansion in the coding region of poly(A) binding protein nuclear 1 (PABPN1). Heterozygous PABPN1 mutant carriers display myopathic symptoms: proximal limb weakness, dysphagia, and ptosis (Davies, Berger, and Rubinsztein 2006). However, individuals with homozygous mutations often display neurological disturbances: cognitive decline, depression, and psychosis (Blumen et al. 2009). The GCG expansion is translated into an expanded poly-alanine tract, which results in the nuclear aggregation of PABPN1 (Davies, Berger, and Rubinsztein 2006). The aggregation of PABPN1 into filamentous nuclear inclusions correlates with retention of large amounts of poly-A containing mRNA (Calado et al. 2000). This suggests that multiple mRNAs may be affected by the mutation in a single gene and fits into a paradigm for diseases caused by malfunctioning of RNA binding proteins. 


\subsection{The multiple steps of RNA processing downstream of transcription allow for multiple regulations and potential errors}

Interactions between mRNA and protein, and mRNA and RNA are responsible for executing distinct steps of post-transcriptional processing on particular RNAs. Most RNA binding proteins and ncRNAs influence the fate of multiple transcripts and therefore problems with either an RNA binding protein or an ncRNA will affect multiple messages. RNA binding proteins can interact with RNA and other proteins. An RNA binding protein has stabilizing and destabilizing effects on target RNAs, depending upon its interactions with other proteins. Figure 3 depicts a hypothetical mRNA with common features indicated and the positions/features where RNA binding proteins and ncRNAs likely bind. A cytoplasmic complex of RNA and proteins is referred to as ribonucleoprotein particle (RNP) and distinctions between types of RNPs are made based upon the protein constituents of different complexes and the fate of transcripts within an RNP. There are many different types of RNPs, but for brevity sake we will simplify the discussion to transport RNPs, Pbodies, and stress granules. Their functions and consequences of their dysfunction as they relate to neurodegenerative diseases will be described in the following sections.

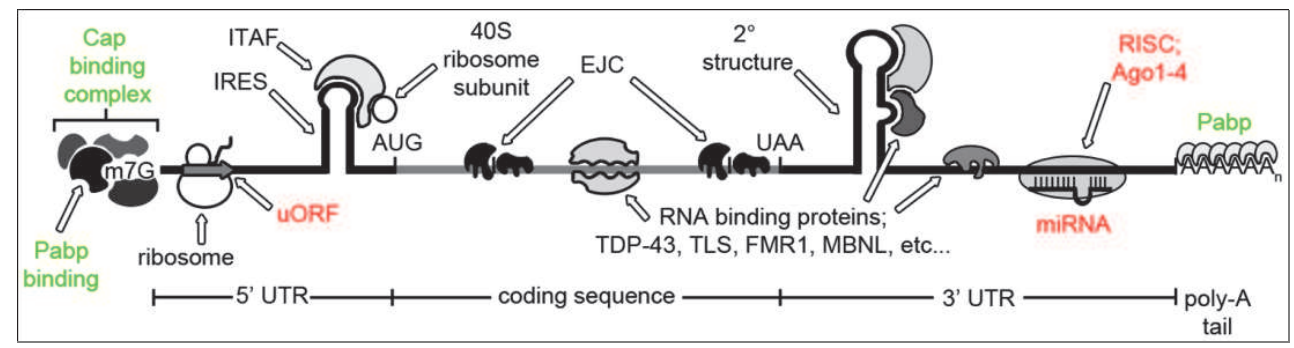

Fig. 3. mRNA processing is mediated by multiple proteins and ncRNAs. The cartoon shows a hypothetical mRNA organized by regions: 5' UTR and 3' UTR (black line segments) and coding sequence (gray line segment). Proteins/entities labeled in green in general have a positive effect on mRNA translation, while those in red tend to inhibit protein translation or mRNA stability. Proteins in black may have positive or negative effects on mRNA translation or stability. The abbreviations used are: m7G (7-methylguanosine), uORF (upstream open reading frame), IRES (internal ribosome entry site), ITAF (IRES-transacting factor), EJC (exonexon junction complex), miRNA (micro RNA), and RISC (RNA-induced silencing complex).

\subsubsection{Transport RNPs deliver mRNAs to discrete locations within a cell}

RNA transport is an efficient means to spatially control gene expression patterns in a single cell. In transporting an mRNA a cell is able to produce multiple protein copies from a single molecule of mRNA at a discrete location, and thus reduce the energy expenditure that would be required if individual protein molecules were instead transported. This mechanism also eliminates the need to suppress the activity of a protein with properties that could be detrimental if present in an inappropriate context (Andreassi and Riccio 2009). Transported mRNAs are translationally repressed until they are delivered to their final destinations by protein- and ncRNA-dependent mechanisms (Wang, Martin, and Zukin 2010). This section will focus on transport RNPs in neurons. 
RNA transport is mediated by specific proteins and/or ncRNAs that function to tether the mRNA to a motor protein complex (Wang, Martin, and Zukin 2010). Movement of transport RNPs out of the soma occurs largely on the backs of the microtubule-based motor proteins kinesin and dynein (Wang, Martin, and Zukin 2010). The microtubules present in axons are all oriented with their plus ends pointed away from the cell interior, and so only the activity of plus-end directed kinesin motors can move cargoes into axons. In contrast, dendrites have a mixed polarity of microtubules in proximal segments; therefore, minus-end directed dynein motors are capable of directing cargoes into dendrites in addition to plus-end directed kinesin motors (Kapitein et al. 2010). Actin-based myosin motors also contribute to mRNA transport and are most likely involved in moving mRNAs from larger bore dendritic chambers into the smaller diameter dendritic spines where actin filaments predominate (Hirokawa, Niwa, and Tanaka 2010).

mRNAs bound for transport are first recognized in the nucleus by trans-acting factors that recognize specific sequence elements present within the mRNA. Although 3' UTR sequence seems a prime candidate for the placement of these location-defining elements, they may also occur within the coding sequence of an mRNA. The protein-bound mRNA is then exported from the nucleus where additional proteins can be recruited to the transcript through binding sites on the trans-acting factor or mRNA (Sossin and DesGroseillers 2006). These RNPs are then thought recognized by motor protein complexes, which then move the mRNA to an appropriate location in the cell.

Fragile $X$ mental retardation protein (FMR1) is an RNA binding protein important for the transport and localization of specific RNAs. FMR1 binds to distinct RNAs and represses their translation, through either a direct influence on the processivity of bound ribosomes and/or an association with Argonaute proteins and miRNAs (De Rubeis and Bagni 2010; Muddashetty et al. 2011). Fragile $X$ syndrome is an inherited intellectual disability that results from a loss of FMR1 expression and is therefore predicted to affect the trafficking and translation of the RNAs normally bound by FMR1 (De Rubeis and Bagni 2010). Many of the RNAs bound by FMR1 are involved in pre- and postsynaptic functions, which suggest that loss of FMR1 may drastically impair neuronal function (Darnell et al. 2011). Interestingly, Huntington's disease protein huntingtin was identified as an FMR1-associated RNA in this study. Staufen is another protein involved in RNA transport and was originally identified in Drosophila, where mutations were shown to affect the asymmetric localization of mRNAs important for embryonic axis generation (St Johnston, Beuchle, and Nusslein-Volhard 1991). A human homolog of Staufen (hStau) co-purifies and co-localizes with RNA, FMR1, kinesin, dynein, and myosin proteins in human cell (Villace, Marion, and Ortin 2004). This example illustrates the combinatorial control and potential redundancy that is utilized for RNA transport.

Once delivered to their final destinations, transported RNAs could be tethered to the cytoskeleton through binding to cytoskeletal associated proteins with affinity for particular mRNAs, or proteins present in the transport RNPs (Kim and Coulombe 2010). Cytoplasmic fractions of cultured cells are enriched for certain mitochondrial RNAs and ribosomal protein RNAs (Russo et al. 2008). Furthermore, disassembly of the microtubule cytoskeleton by nocodazole treatment results in shifting of these mRNAs into the soluble portion of the cytoplasm. A definitive identification of the proteins responsible for cytoskeletal tethering of mRNAs in neurons has not yet been made, although a tug-of-war between different 
polarity-directed motors could effectively produce a localized mRNA. Cytoplasmic FMR1interacting proteins 1 and 2 (CYFIP1, 2) interact with FMR1 and are involved in actin cytoskeletal remodeling (Anitei et al. 2010). Although it has yet to be shown if CYFIP1 or 2 act to anchor FMR1-associated RNAs to the cytoskeleton, this seems like a possibility based on the proteins' affinity for FMR1 and actin remodeling proteins.

Localized transcripts are translationally silenced until the repression is relieved by the dissociation of the inhibitory factor(s). PSD-95 is a post-synaptic scaffolding protein that functions in the regulation of AMPA-type glutamate receptor endocytosis and in the maintenance of dendritic spine architecture. FMR1 along with miR125a (microRNA 125a) and Ago2 bind to the PSD-95 transcript to suppress its translation. The binding of these molecules to the PSD-95 mRNA is dependent upon the phosphorylation of FMR1. When FMR1 is dephosphorylated following group I metabotropic glutamate receptor stimulation, miR125a and Ago2 dissociate from the $3^{\prime}$ UTR of PSD-95 and the mRNA is translated (Muddashetty et al. 2011). This example illustrates how post-translational modifications of RNA binding proteins control their inhibitory or stimulatory effects on mRNA translation.

\subsubsection{P-bodies control mRNA stability}

Processing bodies (P-bodies) share components with transport RNPs, and considerable grey area exists in discriminating between the two types of particles based on protein associations. P-bodies are functionally defined as constitutively present cytoplasmic outposts of RNA degradation or storage (Buchan and Parker 2009). In some instances, mRNAs may be rescued from P-body association and be translated (Brengues, Teixeira, and Parker 2005), although the mechanism for this alternative fate is unclear. P-bodies contain the ribonucleases responsible for $5^{\prime}$ m7G cap removal, $5^{\prime}$ exoribonucleases, deadenylases, as well as components of the RISC pathway (Parker and Sheth 2007).

\subsubsection{Stress granules are large ribonucleoprotein particles that assemble in response to various cellular stressors}

Cellular stress caused by a variety of factors results in the phosphorylation of eIF2A and the subsequent translational silencing of mRNAs not involved in the stress response (Spriggs, Bushell, and Willis 2010). Translationally silenced mRNAs are sequestered away from large ribosomal subunits and the potentially damaging cytoplasmic environment by recruitment into large assemblies of proteins and RNAs called stress granules. Stress granules contain translation initiation factors, small ribosomal subunits, specific RNA binding proteins and their associated RNAs, and general stress granule assembly factors (Buchan and Parker 2009). Experiments in yeast demonstrated that stress granules are dependent upon P-bodies for their assembly, but P-bodies can form when stress granule assembly is inhibited by genetic means (Buchan, Muhlrad, and Parker 2008). mRNAs stored in stress granules can be transferred to P-bodies for degradation and vice-versa (Buchan and Parker 2009). The signals that promote this switch have yet to be identified.

Stress granules form in part through self-association properties, similar to what takes place during protein aggregation. Indeed, several P-body and stress granule associated proteins with glutamine- and asparagine- $(\mathrm{Q} / \mathrm{N})$ rich regions depend on these regions for the self assembly requisite for stress granule and P-body formation (Buchan and Parker 
2009). TDP-43 localizes to stress granules and C-terminal cleavage products generated by caspase 3 are prone to cytoplasmic aggregation (Liu-Yesucevitz et al. 2010). In the event that TDP-43 levels are elevated and there is increased production of C-terminal caspase 3 cleavage products, then this could lead to the nucleation of constitutive stress granules. These stress granules may contain TDP-43 target mRNAs and therefore reduce the levels of the proteins encoded in these mRNAs. By another conspicuous coincidence, caspase 3 is known to cleave mutant huntingtin, which also has intrinsic aggregation properties (Wellington et al. 1998).

Mutations in TLS are associated with a subset of familial cases of ALS (Lagier-Tourenne, Polymenidou, and Cleveland 2010). When these mutant alleles of TLS are expressed in HeLa cells, they localize to stress granules in the absence of added cellular stress (Bosco et al. 2010). Endogenous TLS is also found in stress granules, but only upon experimentally induced cellular stress. These mutant TLS-induced stress granules did not recruit endogenous TLS or TDP-43 into these abnormal structures. If different RNA binding proteins are responsible for delivering their RNA targets to stress granules upon induction of the stress response, then the absence of endogenous TDP-43 from the mutant TLSinduced stress granules could mean that TDP-43 specific mRNAs are also absent from these aggregates.

FMR1 is a well-established stress granule marker and seems to be required for stress granule assembly in mouse and human cells in culture (Didiot et al. 2009). Mouse embryonic fibroblasts from FMR1 null mice do not assemble stress granules, yet P-body assembly is unaffected. FMR1 protein levels increase in response to stress (Didiot et al. 2009). There are currently no mutants in FMR1 that can separate its role in stress granule assembly from its role in transport RNPs, and these structures may in fact be inextricably linked (Kiebler and Bassell 2006). It would be interesting to determine if Fragile $X$ syndrome results from impairment in stress granule assembly or from a reduction in RNA transport, or a combination of the two.

\section{Huntington's disease may involve detrimental changes in post- transcriptional gene expression patterns}

There is mounting circumstantial and experimental evidence implicating deviations in posttranscriptional RNA processing events in the establishment and/or progression of HD. Changes in post-transcriptional processing of RNA could account for the enhanced susceptibility of certain types of cells in HD if specific RNAs important for the survival or proper functioning of these cells are affected by mutant huntingtin (Htt) expression. Here we will review the evidence that suggests $\mathrm{Htt}$ is normally involved in RNA processing, and mutant Htt expression may impair normal RNA processing or activate stress responses that alters gene expression.

\subsection{Huntingtin association with Ago2 is important for RNA silencing pathway}

Our group discovered an Htt-Ago2 association through affinity purification and mass spectrometry of a FLAG-tagged N-terminal fragments of wild-type (25 glutamines) and mutant (97 glutamines) Htt expressed in HeLa cells (Savas et al. 2008). We went on to show that endogenous Htt co-localizes with Ago2 and Dcp1 at P-bodies in U2OS cells. 
Because these $\mathrm{N}$-terminal Htt purifications did not contain dicer or other proteins involved in the biogenesis of miRNAs, we hypothesized that Htt was involved in the effector stage of the RNA silencing pathway. Knockdown of Htt in U2OS cells by RNAi revealed that a subset of P-bodies required Htt for their assembly. Incomplete inhibition of P-body assembly could have resulted from incomplete knockdown of Htt. Alternatively, a specific subset of RNPs could require Htt for their incorporation into functional P-bodies. Interestingly, a knock-in striatal precursor cell line expressing mutant Htt (Trettel et al. 2000) formed fewer P-bodies than a wild-type striatal precursor cell line (Savas et al. 2008). This observation suggests that mutant Htt may have a dominant negative or loss-of-function effect on the RNA silencing pathway. We further demonstrated that knockdown of Htt inhibits the RNA interference response by reporter assays, and that mutant Htt-expressing cells are less efficient in this pathway. Finally, fluorescence recovery after photobleaching (FRAP) analysis of GFP-Ago2 dynamics in wild-type (25 glutamines) and mutant (97 glutamines) Htt fragment-expressing cells demonstrated that mutant Htt inhibits the recruitment of Ago2 to P-bodies.

In a follow-up study, we focused our analysis on cultured cortical neurons and a neuroblastoma cell line. This work showed that Htt co-localizes, co-fractionates, and copurifies with Ago2 in cultured cortical neurons (Savas et al. 2010). We also discovered that tethering $\mathrm{Htt}$ to a luciferase reporter mRNA through a $\lambda \mathrm{N}$ element-box-B interaction represses luciferase expression. This repression was found to be at least partially dependent on the presence of Ago2 in the assay. In summary, the Ago2-Htt interaction uncovered by affinity purification has been verified by numerous assays and is functionally important in the RNA silencing pathway. Moreover, mutant Htt-expressing cells seem to be less able to utilize this pathway, possibly through a decreased recruitment of Ago2 to P-bodies. We have since found that full-length FLAG-tagged mutant Htt expressed from the endogenous locus associates with Ago2 in mouse brains (Culver, Savas, et. al. submitted). Based on these observations we suggest that Htt may contribute to the silencing of a specific subset of messages and that mutant Htt interfering with this process could give rise to inappropriate levels of particular target proteins.

\subsection{Huntingtin contributes to mRNA transport in neurons}

Our studies indicate that Htt co-localizes with mRNAs, and lentiviral-mediated knockdown of $\mathrm{Htt}$ drastically reduces the number of punctate polyadenylated RNA-containing particles detected by FISH in cultured cortical neurons. Furthermore, Htt co-localizes with Staufen and co-traffics with an MS2-tagged $\mathrm{IP}_{3} \mathrm{R} 1$ 3' UTR (inositol 1,4,5-trisphosphate receptor 1) mRNA in cultured cortical neurons (Savas et al. 2010). Htt is known to associate with the microtubule-based motor dynein complex (Caviston et al. 2007). These data seem to suggest that Htt may be required for all directed dendritic RNA transport in cultured neurons. Although we did not demonstrate that mutant Htt expression had any effect on this process, based on the inhibitory effect that mutant Htt expression has on vesicular transport of BDNF- and APP-containing vesicles (Gauthier et al. 2004; Her and Goldstein 2008), it seems likely that mutant Htt will also inhibit RNA transport. If this is the case, then many mRNAs may be inappropriately localized and neurons could be less able to respond to stimuli through local translation. 
BDNF levels are reduced in HD patient brains and exogenous delivery of BDNF rescues many of the phenotypes in a mouse model of HD (Gharami et al. 2008). Furthermore, mutant Htt expression inhibits the trafficking of BDNF-containing vesicles in cultured cortical neurons (Gauthier et al. 2004). We have recently found that Htt co-localizes with BDNF mRNA in cultured cortical neurons and in brain cortical sections (Ma et al. 2010). These observations suggest that not only Htt is important for BDNF protein trafficking, but also mRNA trafficking. It is also interesting that wild-type Htt overexpression increases BDNF mRNA levels, while mutant Htt overexpression reduces BDNF mRNA. Furthermore, BDNF mRNA levels are reduced in HD brain compared with unaffected individuals (Zuccato et al. 2001). It would therefore seem that Htt influences BDNF levels and location from transcription to mRNA localization, to delivery of the translated protein to its sites of action.

\subsection{Mutant Htt aggregates may cause RNA processing defects through sequestration of RNA binding proteins}

Cytoplasmic Htt-containing aggregates in HD brain tissue contain the RNA binding proteins TDP-43 and TLS (Doi et al. 2010; Schwab et al. 2008). Intriguingly, these same two RNA binding proteins also form aggregates in ALS and FTLD (a type of dementia). TDP-43 is a widely expressed RNA binding protein with roles in RNA splicing, stability, and regulation of protein translation. TDP-43 purifications from UV cross-linked sources identified thousands of mRNAs bound by TDP-43. Many of the RNAs were involved in RNA metabolic processes, and Htt was among the list of mRNAs that co-purified with cross-linked TDP-43 (Sephton et al. 2011). TDP-43 also binds to its own mRNA and leads to its degradation (Ayala et al. 2011). This finding, along with the findings discussed in section 2.2.2, suggest that TDP-43 activity is important for controlling the levels of particular transcripts. Over-expression of TDP-43 is sufficient to induce neurodegenerative phenotypes in model organisms (Ash et al. 2010; Tatom et al. 2009). Therefore, a reduced capacity of TDP-43 to regulate its own mRNA levels through sequestration of the protein in aggregates could produce increasing levels of TDP-43 available for aggregation and thus accelerate toxicity.

TLS is similar to TDP-43 in that it is involved in many different steps of RNA processing, from transcription and RNA splicing, to mRNA stability, and transport. It is also similar to TDP-43 in that it forms aggregates in ALS in cases where mutations in TLS correlate with disease presentation (Lagier-Tourenne, Polymenidou, and Cleveland 2010). TDP-43 and TLS aggregation seem to be mutually exclusive, as TDP-43 aggregations were not observed in ALS cases associated with mutations in TLS (Vance et al. 2009). This observation strongly argues that alterations in RNA processing events can cause ALS and FTLD by multiple independent means. Clinical presentations of HD are somewhat heterogeneous: some patients display more severe forms of psychiatric and intellectual disturbances, as well as varying degrees of mobility impairment. These differences in clinical presentation are not correlated with differences in CAG repeat lengths (Weigell-Weber, Schmid, and Spiegel 1996), which suggests that additional genetic or environmental factors contribute to the differences seen in HD patients. We hypothesize that these differences could be accounted for by unique combinations of alterations in the post-transcriptional processing of specific RNAs. 


\subsection{Htt associates with proteins involved in RNA splicing and cleavage}

A yeast two-hybrid screen using the N-terminus of Htt identified three RNA binding proteins that interacted with Htt. Two of these proteins are involved in RNA splicing activity and are widely conserved in eukaryotic evolution (PRPF40A and PRPF40B). They are both general components of the core splicing machinery and as such, would be predicted to have broad effects if their activity were perturbed (Faber et al. 1998). As would be expected of a protein involved in mRNA splicing, PRPF40A is predominantly a nuclear protein. However, the protein is redirected to the cytoplasm when co-overexpressed with a mutant form of a Htt fragment (Jiang et al. 2011). Furthermore, mutant Htt fragments more strongly interact with PRPF40A than wild-type fragments. The authors suggest that mutant Htt may actively sequester PRPF40A in the cytoplasm and thereby inhibit the protein's normal splicing activity.

The other RNA binding protein identified in this yeast two-hybrid screen is known as symplekin (SYMPK) and is involved in polyadenylation of mRNAs. SYMPK is present in a large complex containing other proteins involved in mRNA cleavage and polyadenylation (Kolev and Steitz 2005). Although SYMPK was initially identified by yeast two-hybrid, the protein was also present along with other members of the cleavage and polyadenylation specificity factor (CPSF) complex in Htt purifications from HeLa cells (our unpublished observations). SYMPK is required for both nuclear and cytoplasmic polyadenylation events (Barnard et al. 2004). Therefore, any changes in SYMPK activity could have a global impact on the stability and translational potential of mRNAs. Interestingly however, SYMPK was identified in a genome-wide screen for modifiers of mitotic fidelity (Cappell et al. 2010). The influence of SYMPK on spindle positioning was shown to occur through its role in polyadenylation, as knockdown of other genes involved in this process produced a similar effect. It is therefore possible that specific cellular processes are more sensitive to a reduction in polyadenylated transcripts than others.

\subsection{Mutant Htt mRNA may have detrimental effects independent of coding for mutant Htt protein}

Mutant Htt mRNA can form hairpin loops through G-C base pairing amongst the CAG repeats and the downstream CGG repeats of the poly-proline encoding region (de Mezer et al. 2011). These hairpins are bound by the double-stranded RNA dependent protein kinase PKR (Peel et al. 2001). Furthermore, PKR activity is elevated in HD patient brain tissue (Bando et al. 2005). PKR is activated by viral infection and acts to repress $5^{\prime} \mathrm{m} 7 \mathrm{G}$ capdependent translation of endogenous transcripts through phosphorylation of eIF2A (Spriggs, Bushell, and Willis 2010). Activated PKR induces the stress response and preferentially allows for the translation of mRNAs containing an IRES element immediately upstream of the initiation codon. Htt contains a uORF in its 5' UTR, which can inhibit the translation of a CAT reporter when fused upstream of the CAT ATG codon (Lee et al. 2002). This $\mathrm{uORF}$ is predicted to reduce the translation of Htt under normal conditions when capdependent translation is employed. The intervening sequence between the $\mathrm{uORF}$ in the Htt 5' UTR and the initiation codon of Htt is GC-rich. Furthermore, this sequence is predicted to form a long hairpin structure by the RNAfold webserver sequence analysis program. In the future it would be interesting to determine if $\mathrm{Htt}$ can be translated in the absence of a $5^{\prime}$ m7G cap. 
The ability of CAG-expanded mRNA to form extended hairpin loops leaves open the possibility that the mRNA could act as a molecular sponge for other RNA binding proteins and produce a toxic response similar to what has been proposed for myotonic dystrophy type 1 (DM1) and fragile $X$ tremor ataxia syndrome (FXTAS). In this model, mutant Htt mRNA could actively recruit RNA binding proteins that recognize the CAG/CGG hairpin, and either prevents the RNA binding proteins from performing their normal activities through an effective reduction in their levels, or initiates an inappropriate response through a scaffolding-type effect between different RNA binding proteins such as PKR. We have noticed that mutant Htt knock-in cell lines and animals produce less mutant protein than wild-type, despite identical $5^{\prime}$ regions (unpublished observations). The expanded CAG region could therefore inhibit the translation of mutant Htt protein through the CAG/CGG hairpin structure.

CNG (N represents any nucleotide) expanded mRNAs were demonstrated to lead to translation initiation independent of an initiation codon ( $\mathrm{Zu}$ et al. 2011). This ATGindependent translation occurred only when the extent of expansion was beyond a certain threshold. Strikingly, the threshold for CAG expansion necessary to produce ATG independent translation occurred at 42 CAG repeats, which is remarkably close to the threshold for HD diagnosis (Group 1993). This exciting finding raises the possibility that mutant $\mathrm{Htt}$ may be translated by a $5^{\prime}$ m7G cap-independent mechanism. Furthermore, these authors ( $\mathrm{Zu}$ et al. 2011) showed that the CAG-expanded RNA was capable of producing protein in all three frames of translation, meaning that poly-alanine, poly-serine, and polyglutamine proteins would be produced from this expansion. In SCA3 (spinocerebellar ataxia type 3), CAG repeat expansion also produces a poly-alanine peptide in addition to the polyglutamine peptide (Gaspar et al. 2000). Similarly, poly-alanine and poly-serine proteins were found in HD patient brain tissues where they localized to ubiquitin-positive intranuclear inclusions (Davies and Rubinsztein 2006). Poly-alanine expansion in PABPN1 results in nuclear aggregation of the protein in oculopharyngeal muscular dystrophy (Davies, Berger, and Rubinsztein 2006). Poly-alanine or poly-serine containing peptides produced from frame-shifted translation of Htt would encounter stop codons very shortly after the homopolymeric stretch of amino acids. This would result in the production of very small proteins, which could diffuse through the nuclear pore without the aid of the import machinery or a nuclear localization element. Poly-alanine stretches are highly hydrophobic and are predicted to form stable and compact $\beta$-sheets, which are assembled into insoluble fibrils as the lowest energy conformation (Shinchuk et al. 2005).

\subsection{Mutant Htt purifications from mouse brain are enriched in RNA binding proteins and protein translation machinery components}

We have recently used affinity purification and mass spectrometry to identify the cellular pathways most likely affected by mutant Htt expression in mouse brains. Our as yet unpublished observations demonstrated that mutant Htt associates with vast numbers of proteins involved in translation initiation and RNA metabolic processes. These functional categories were significantly better represented in mutant purifications than wild-type, which argues that mutant $\mathrm{Htt}$ disproportionately affects these processes compared with wild-type. We also found that mutant Htt expression influenced the solubility of two of its newly identified interaction partners, FMR1 and PABP by affecting their sensitivity to 
treatment with RNAse. These observations suggest that mutant Htt expression affects the activity of at least two RNA binding proteins known to target many mRNAs.

One of the commonalities between the many RNA binding proteins and translational proteins identified in our purifications was their known involvement in stress granule assembly or function. This led us to discover that both wild-type and mutant Htt localize to stress granules (Culver, Savas, et. al, submitted). Similar to what we previously observed with regards to mutant Htt expression on P-body formation (Savas et al. 2008), we found that mutant Htt-expressing striatal precursor cell line (Trettel et al. 2000) formed fewer, but larger stress granules than a wild-type version of these cells (Culver, Savas, et. al, submitted). Htt is not required for stress granule assembly, however, as Htt-null mouse ES cells are able to form stress granules as well as wild-type cells (our unpublished observation). Based on these data, we propose that Htt may help to deliver specific mRNAs to stress granules, but is not required for their assembly.

Late stage HD patient brains exhibit reduced electron transport chain activity in mitochondrial complex II, III, and IV when assayed postmortem (Gu et al. 1996). In addition, systemic delivery of the complex II inhibitor 3-nitropropionic acid results in striatal-specific cell death in rodents and non-human primates (Brouillet et al. 2005). Inhibition of the electron transport chain can lead to accumulation of reactive oxygen species (ROS) (Chen et al. 2007). ROS species can activate the stress response, lead to the formation of stress granules, and subsequently suppress the translation of many different kinds of proteins. Since the striatum seems to be exquisitely sensitive to electron transport chain inhibition, perhaps the death of the cells within this tissue stems from a reduced ability to synthesize the proteins required for maintaining neuronal activity and viability. This hypothesis agrees with the increased levels of activated PKR seen in HD patient brain tissue as mentioned above. Indeed, ROS generation has been shown to increase PKR transcription and hence activity of the kinase (Pyo, Lee, and Choi 2008). We speculate that cellular stress, either owing to mutant Htt expression, or normally experienced by the brain, acts to activate the stress response, which results in a chronic down regulation of translation of proteins vital to the integrity of the neurons of the striatum. A similar type of process may also contribute to the neurodegeneration accompanying a stroke or the chronic head injuries experienced by athletes involved in contact sports.

Mutant Htt aggregates in HD contain RNA binding proteins (Doi et al. 2010; Schwab et al. 2008) and we have since found that expression of an aggregate-prone fragment of mutant $\mathrm{Htt}$ in mouse neuroblastoma cell line N2A produces mutant Htt aggregates that also contain RNA (Culver, Savas et. al, submitted). It is therefore possible that the mutant Htt aggregates present in HD also contain RNA. There are two possible interpretations for the aggregation of RNA binding proteins in neurodegenerative diseases that are not mutually exclusive. One is that that the aggregation of RNA binding proteins prevents them from acting on their normal targets, thereby altering the expression patterns of particular RNAs. It is also possible that certain RNAs are also sequestered within protein aggregates and this sequestration prevents the translation of these RNAs. One could imagine that disease-bydisease combinations of aggregated RNA binding proteins and RNAs could produce the defining symptoms and features for a particular disease. These defining features would depend on the combinations of genes affected in each disease. 


\subsection{Htt protein associates with its own mRNA}

One of the necessary conditions for the hypothesis that mutant Htt influences posttranscriptional gene expression is that there must be specific mRNAs adversely affected over others. It seems unlikely that a global interference with this process by mutant $\mathrm{Htt}$ would produce the specific effects of the disease. We have attempted to identify these specific mRNAs through affinity purification and microarray profiling of Htt-associated RNAs in mouse brains. In agreement with the results of our mass spectrometry data, we have found that mutant Htt purifications contained substantially more enriched mRNAs than wild-type purifications (unpublished observations). Although, many of these enriched mRNAs have been reproduced and have direct relevance to HD, the identifications are still too preliminary to be reported here. However, we are confident that Htt protein purifications reproducibly recovered substantial amounts of Htt mRNA. Despite reduced amounts of mutant Htt protein recovery, these purifications contained substantially more Htt mRNA than wild-type purifications (Figure 4).
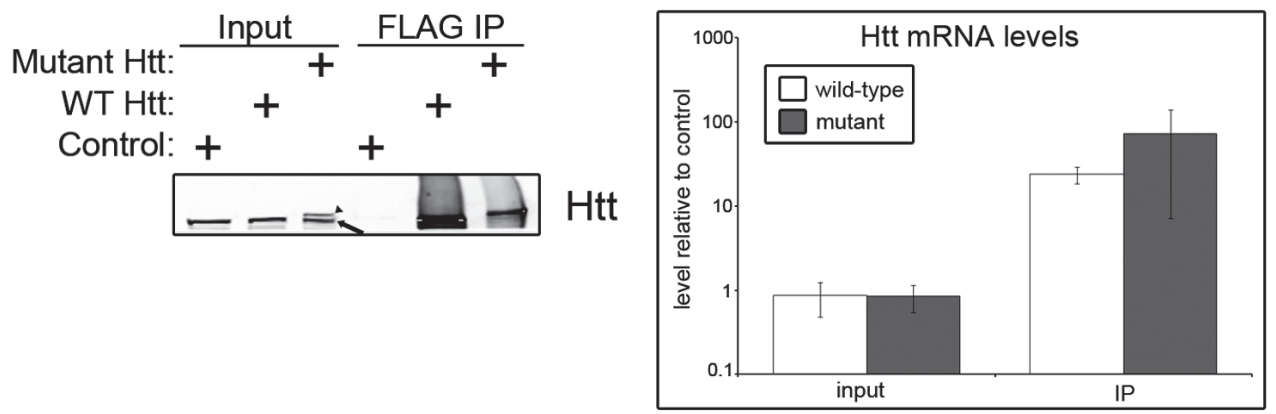

Fig. 4. Mutant Htt associates with more of its own mRNA than wild-type in mouse brains. The western blot on the left shows that wild-type Htt purifications recover more protein than mutant purifications. Htt was recovered (FLAG IP) from a cytoplasmic fraction of FLAG-tagged wild-type and mutant Htt knock-in mouse brains. Control purifications were from non-transgenic littermates. The blot is probed with an anti-Htt antibody. The bar graph on the right shows the levels of Htt mRNA in the inputs and affinity purifications (IP) from three independent experiments relative to the control.

This result has since been reproduced in cultured neurons and neuroblastoma cell lines using an Htt specific antibody (Culver, unpublished observations). This unexpected and exciting finding has interesting parallels to TDP-43 autoregulation of its own mRNA, and our lab is currently investigating if a similar phenomenon occurs with respect to Htt and its own mRNA.

\section{Conclusion}

There is now considerable evidence that Htt influences multiple steps of post-transcriptional gene expression. Htt interacts with proteins involved in RNA splicing, and expression of a mutant $\mathrm{Htt}$ fragment results in the cytoplasmic retention of a pre-mRNA processing factor 
PRPF40a. Splicing occurs in the nucleus and therefore the absence of a splicing factor from here is functionally equivalent to a loss-of-function of the protein. This could have far reaching consequences in cases where redundancy mechanisms preclude splicing activity rescue for PRPF40a. The advent of highly sensitive RNA sequencing technology could be used to identify small or subtle differences in the splicing patterns of mRNAs in HD compared with an unaffected population. Potential differences in splicing patterns could be informative in characterizing how HD pathology progresses. Ideally, we would like to see a link between affected mRNAs and splicing proteins known to associate with $\mathrm{Htt}$.

Htt interacts with Ago2, localizes to P-bodies, and participates in RNA silencing. Furthermore, mutant Htt-expressing cells possess fewer P-bodies and less efficiently execute the RNA silencing response. Artificial tethering of Htt to a luciferase reporter reduces its expression and this inhibition is partially mediated by Ago2. If the RNA silencing response is less effective in HD, then this could lead to increased levels of mRNAs and proteins whose levels are normally tightly controlled. This key step in regulation of gene expression is critical to ensure that potentially damaging gene products are only expressed under the right conditions. An impressive microarray study of HD patients and unaffected individuals has already pinpointed several mRNAs whose levels are elevated in HD compared with unaffected individuals (Hodges et al. 2006). In the future it will be important to determine if any of these mRNAs is regulated post-transcriptionally by Argonaute proteins and the RNA silencing pathway.

Mutant Htt mRNA can form hairpin structures composed of CAG and neighboring CGG repeats. These hairpins are recognized by the double stranded RNA binding protein kinase PKR, which acts to repress translation by inhibiting $\mathrm{m} 7 \mathrm{G}$ cap-dependent translation through phosphorylation of eIF2A. PKR levels are elevated in HD patient brain tissue and elevated PKR activity can induce the formation of stress granules through global m7G cap-dependent translational inhibition. Expression of a CUG-expanded RNA is sufficient to induce stress granule formation in a cell culture model of myotonic dystrophy type I. This response is mediated by PKR activation (Huichalaf et al. 2010). These stress granules sequester an mRNA encoding a key DNA repair enzyme. Both wild-type and mutant Htt localize to stress granules, although Htt seems to be dispensable for their formation. Aggregation of proteins required for stress granule and P-body assembly involves glutamine-rich regions. We propose that stress granules are assembled in Htt either through activation of PKR by expanded CAG hairpins, increased ROS generation through electron transport chain impairment, or a combination of both processes. Htt localization to stress granules could lead to increased recruitment of Htt to stress granules through the expanded polyglutamine repeat sequence of mutant Htt. In this model, stress granules therefore serve as a nucleating factor in Htt aggregate assembly. The RNAs that are trapped within these stress granulenucleated aggregates will therefore be prevented from being translated. In theory this could generate a feedback loop which leads to increased accumulation of Htt as the stress response is perpetuated. In cell culture, mutant Htt aggregates contain RNA. In the future we would like to identify the specific RNAs that may be trapped within insoluble Htt aggregates as they could be key factors in determining HD disease symptoms.

Htt is involved in trafficking RNAs in cultured neurons. Lentiviral-mediated Htt knockdown in cultured cortical neurons results in a drastic reduction in the amount of large ribonucleoprotein particles containing polyadenylated RNA present in dendrites. Htt co- 
localizes with proteins important for transporting RNAs and with the BDNF mRNA in rat brain slices and in cultured cortical neurons. Mutant Htt expression inhibits transport of vesicular cargoes and so it is likely that mutant Htt expression will also inhibit RNA transport by a similar mechanism. Localized protein translation is known to be important for synaptic plasticity. If mutant Htt reduces the amount of RNA transported, then this could manifest as a decreased ability of cells to quickly respond to changes in their environment. Data from our lab suggests that Htt plays a central role in RNA transport, with little specificity for particular mRNAs. Further experiments in simpler model systems (e.g. flies) will be required to determine if this is in fact the case. If defects in systemic RNA transport contribute to $\mathrm{HD}$, then genetic studies in mouse on genes with central roles in RNA transport should produce a similar constellation of phenotypes as HD.

If HD results in part from the post-transcriptional deregulation of specific RNAs, then these transcripts will need to be identified to strengthen this hypothesis. These genes should function in a process that is known to be perturbed in HD or be important for the proper functioning or survival of striatal and cortical neurons. We are currently identifying Httassociated RNAs by affinity purification of wild-type and mutant Htt from mouse brains. The significance of these potential interactions will be verified in animal models and human tissue. It is our hope that these experiments will further our understanding of HD and possibly contribute to treatment and a cure.

\section{References}

An, J. J., et al. 2008. Distinct role of long 3' UTR BDNF mRNA in spine morphology and synaptic plasticity in hippocampal neurons. Cell 134 (1):175-87.

Andreadis, A. 2011. Tau splicing and the intricacies of dementia. Journal of cellular physiology.

Andreassi, C., \& A. Riccio. 2009. To localize or not to localize: mRNA fate is in 3'UTR ends. Trends in cell biology 19 (9):465-74.

Anitei, M., et al. 2010. Protein complexes containing CYFIP/Sra/PIR121 coordinate Arf1 and Rac1 signalling during clathrin-AP-1-coated carrier biogenesis at the TGN. Nature cell biology 12 (4):330-40.

Ash, P. E., et al. 2010. Neurotoxic effects of TDP-43 overexpression in C. elegans. Human molecular genetics 19 (16):3206-18.

Ayala, Y. M., et al. 2011. TDP-43 regulates its mRNA levels through a negative feedback loop. The EMBO journal 30 (2):277-88.

Bando, Y., et al. 2005. Double-strand RNA dependent protein kinase (PKR) is involved in the extrastriatal degeneration in Parkinson's disease and Huntington's disease. Neurochemistry international 46 (1):11-8.

Barnard, D. C., et al. 2004. Symplekin and xGLD-2 are required for CPEB-mediated cytoplasmic polyadenylation. Cell 119 (5):641-51.

Blumen, S. C., et al. 2009. Cognitive impairment and reduced life span of oculopharyngeal muscular dystrophy homozygotes. Neurology 73 (8):596-601.

Bosco, D. A., et al. 2010. Mutant FUS proteins that cause amyotrophic lateral sclerosis incorporate into stress granules. Human molecular genetics 19 (21):4160-75.

Brengues, M., D. Teixeira, \& R. Parker. 2005. Movement of eukaryotic mRNAs between polysomes and cytoplasmic processing bodies. Science 310 (5747):486-9. 
Brouillet, E., et al. 2005. 3-Nitropropionic acid: a mitochondrial toxin to uncover physiopathological mechanisms underlying striatal degeneration in Huntington's disease. Journal of neurochemistry 95 (6):1521-40.

Buchan, J. R., D. Muhlrad, \& R. Parker. 2008. P bodies promote stress granule assembly in Saccharomyces cerevisiae. The Journal of cell biology 183 (3):441-55.

Buchan, J. R., \& R. Parker. 2009. Eukaryotic stress granules: the ins and outs of translation. Molecular cell 36 (6):932-41.

Calado, A., et al. 2000. Nuclear inclusions in oculopharyngeal muscular dystrophy consist of poly(A) binding protein 2 aggregates which sequester poly(A) RNA. Human molecular genetics 9 (15):2321-8.

Cappell, K. M., et al. 2010. Symplekin specifies mitotic fidelity by supporting microtubule dynamics. Molecular and cellular biology 30 (21):5135-44.

Caviston, J. P., et al. 2007. Huntingtin facilitates dynein/dynactin-mediated vesicle transport. Proceedings of the National Academy of Sciences of the United States of America 104 (24):10045-50.

Chen, Y., et al. 2007. Mitochondrial electron-transport-chain inhibitors of complexes I and II induce autophagic cell death mediated by reactive oxygen species. Journal of cell science 120 (Pt 23):4155-66.

Darnell, J. C., et al. 2011. FMRP Stalls Ribosomal Translocation on mRNAs Linked to Synaptic Function and Autism. Cell 146 (2):247-61.

Davies, J. E., Z. Berger, \& D. C. Rubinsztein. 2006. Oculopharyngeal muscular dystrophy: potential therapies for an aggregate-associated disorder. The international journal of biochemistry \& cell biology 38 (9):1457-62.

Davies, J. E., \& D. C. Rubinsztein. 2006. Polyalanine and polyserine frameshift products in Huntington's disease. Journal of medical genetics 43 (11):893-6.

de Mezer, M., et al. 2011. Mutant CAG repeats of Huntingtin transcript fold into hairpins, form nuclear foci and are targets for RNA interference. Nucleic acids research 39 (9):3852-63.

De Rubeis, S., \& C. Bagni. 2010. Fragile X mental retardation protein control of neuronal mRNA metabolism: Insights into mRNA stability. Molecular and cellular neurosciences 43 (1):43-50.

Didiot, M. C., et al. 2009. Cells lacking the fragile X mental retardation protein (FMRP) have normal RISC activity but exhibit altered stress granule assembly. Molecular biology of the cell 20 (1):428-37.

Doi, H., et al. 2010. The RNA-binding protein FUS/TLS is a common aggregate-interacting protein in polyglutamine diseases. Neuroscience research 66 (1):131-3.

Faber, P. W., et al. 1998. Huntingtin interacts with a family of WW domain proteins. Human molecular genetics 7 (9):1463-74.

Ferrer, I., et al. 2000. Brain-derived neurotrophic factor in Huntington disease. Brain research 866 (1-2):257-61.

Friedman, R. C., et al. 2009. Most mammalian mRNAs are conserved targets of microRNAs. Genome research 19 (1):92-105.

Gaspar, C., et al. 2000. CAG tract of MJD-1 may be prone to frameshifts causing polyalanine accumulation. Human molecular genetics 9 (13):1957-66.

Gasparini, L., B. Terni, \& M. G. Spillantini. 2007. Frontotemporal dementia with tau pathology. Neuro-degenerative diseases 4 (2-3):236-53. 
Gauthier, L. R., et al. 2004. Huntingtin controls neurotrophic support and survival of neurons by enhancing BDNF vesicular transport along microtubules. Cell 118 (1):127-38.

Gharami, K., et al. 2008. Brain-derived neurotrophic factor over-expression in the forebrain ameliorates Huntington's disease phenotypes in mice. Journal of neurochemistry 105 (2):369-79.

Gingras, A. C., B. Raught, \& N. Sonenberg. 1999. eIF4 initiation factors: effectors of mRNA recruitment to ribosomes and regulators of translation. Annual review of biochemistry 68:913-63.

Group, The Huntington's Disease Collaborative Research. 1993. A novel gene containing a trinucleotide repeat that is expanded and unstable on Huntington's disease chromosomes. Cell 72 (6):971-83.

$\mathrm{Gu}, \mathrm{M}$., et al. 1996. Mitochondrial defect in Huntington's disease caudate nucleus. Annals of neurology 39 (3):385-9.

Hebert, S. S., et al. 2010. Genetic ablation of Dicer in adult forebrain neurons results in abnormal tau hyperphosphorylation and neurodegeneration. Human molecular genetics 19 (20):3959-69.

Her, L. S., \& L. S. Goldstein. 2008. Enhanced sensitivity of striatal neurons to axonal transport defects induced by mutant huntingtin. The Journal of neuroscience : the official journal of the Society for Neuroscience 28 (50):13662-72.

Hirokawa, N., S. Niwa, \& Y. Tanaka. 2010. Molecular motors in neurons: transport mechanisms and roles in brain function, development, and disease. Neuron 68 (4):610-38.

Hodges, A., et al. 2006. Regional and cellular gene expression changes in human Huntington's disease brain. Human molecular genetics 15 (6):965-77.

Hughes, T. A. 2006. Regulation of gene expression by alternative untranslated regions. Trends in genetics : TIG 22 (3):119-22.

Huichalaf, C., et al. 2010. Expansion of CUG RNA repeats causes stress and inhibition of translation in myotonic dystrophy 1 (DM1) cells. The FASEB journal : official publication of the Federation of American Societies for Experimental Biology 24 (10):370619.

Jiang, H., et al. 2004. Myotonic dystrophy type 1 is associated with nuclear foci of mutant RNA, sequestration of muscleblind proteins and deregulated alternative splicing in neurons. Human molecular genetics 13 (24):3079-88.

Jiang, Y. J., et al. 2011. Interaction with Polyglutamine-expanded Huntingtin Alters Cellular Distribution and RNA Processing of Huntingtin Yeast Two-hybrid Protein A (HYPA). The Journal of biological chemistry 286 (28):25236-45.

Jin, P., et al. 2003. RNA-mediated neurodegeneration caused by the fragile $X$ premutation rCGG repeats in Drosophila. Neuron 39 (5):739-47.

Kapitein, L. C., et al. 2010. Mixed microtubules steer dynein-driven cargo transport into dendrites. Current biology : CB 20 (4):290-9.

Kiebler, M. A., \& G. J. Bassell. 2006. Neuronal RNA granules: movers and makers. Neuron 51 (6):685-90.

Kim, S., \& P. A. Coulombe. 2010. Emerging role for the cytoskeleton as an organizer and regulator of translation. Nature reviews. Molecular cell biology 11 (1):75-81. 
Kolev, N. G., \& J. A. Steitz. 2005. Symplekin and multiple other polyadenylation factors participate in 3'-end maturation of histone mRNAs. Genes $\mathcal{E}$ development 19 (21):2583-92.

Lagier-Tourenne, C., M. Polymenidou, \& D. W. Cleveland. 2010. TDP-43 and FUS/TLS: emerging roles in RNA processing and neurodegeneration. Human molecular genetics 19 (R1):R46-64.

Lee, J., et al. 2002. An upstream open reading frame impedes translation of the huntingtin gene. Nucleic acids research 30 (23):5110-9.

Lemay, J. F., et al. 2010. Crossing the borders: poly(A)-binding proteins working on both sides of the fence. RNA biology 7 (3):291-5.

Liu-Yesucevitz, L., et al. 2010. Tar DNA binding protein-43 (TDP-43) associates with stress granules: analysis of cultured cells and pathological brain tissue. PLoS One 5 (10):e13250.

Lovett, P. S., \& E. J. Rogers. 1996. Ribosome regulation by the nascent peptide. Microbiological reviews 60 (2):366-85.

Ma, B., et al. 2010. Localization of BDNF mRNA with the Huntington's disease protein in rat brain. Molecular neurodegeneration 5:22.

Mackenzie, I. R., et al. 2007. Pathological TDP-43 distinguishes sporadic amyotrophic lateral sclerosis from amyotrophic lateral sclerosis with SOD1 mutations. Annals of neurology 61 (5):427-34.

Mahadevan, M., et al. 1992. Myotonic dystrophy mutation: an unstable CTG repeat in the $3^{\prime}$ untranslated region of the gene. Science 255 (5049):1253-5.

Mangus, D. A., M. C. Evans, \& A. Jacobson. 2003. Poly(A)-binding proteins: multifunctional scaffolds for the post-transcriptional control of gene expression. Genome biology 4 (7):223.

Mattick, J. S., \& I. V. Makunin. 2006. Non-coding RNA. Human molecular genetics 15 Spec No 1:R17-29.

Meyer, S., C. Temme, \& E. Wahle. 2004. Messenger RNA turnover in eukaryotes: pathways and enzymes. Critical reviews in biochemistry and molecular biology 39 (4):197-216.

Mihailovich, M., et al. 2007. Complex translational regulation of BACE1 involves upstream AUGs and stimulatory elements within the 5 ' untranslated region. Nucleic acids research 35 (9):2975-85.

Muddashetty, R. S., et al. 2011. Reversible inhibition of PSD-95 mRNA translation by miR125a, FMRP phosphorylation, and mGluR signaling. Molecular cell 42 (5):673-88.

Narisawa-Saito, M., et al. 1996. Regional specificity of alterations in NGF, BDNF and NT-3 levels in Alzheimer's disease. Neuroreport 7 (18):2925-8.

Oe, S., \& Y. Yoneda. 2010. Cytoplasmic polyadenylation element-like sequences are involved in dendritic targeting of BDNF mRNA in hippocampal neurons. FEBS letters 584 (15):3424-30.

Parker, R., \& U. Sheth. 2007. P bodies and the control of mRNA translation and degradation. Molecular cell 25 (5):635-46.

Peel, A. L., et al. 2001. Double-stranded RNA-dependent protein kinase, PKR, binds preferentially to Huntington's disease (HD) transcripts and is activated in HD tissue. Human molecular genetics 10 (15):1531-8. 
Peng, S., et al. 2009. Decreased brain-derived neurotrophic factor depends on amyloid aggregation state in transgenic mouse models of Alzheimer's disease. The Journal of neuroscience : the official journal of the Society for Neuroscience 29 (29):9321-9.

Pickering, B. M., \& A. E. Willis. 2005. The implications of structured 5' untranslated regions on translation and disease. Seminars in cell $\mathcal{E}$ developmental biology 16 (1):39-47.

Pritchard, S. M., et al. 2011. The toxicity of tau in Alzheimer disease: turnover, targets and potential therapeutics. Journal of cellular and molecular medicine 15 (8):1621-35.

Pruunsild, P., et al. 2007. Dissecting the human BDNF locus: bidirectional transcription, complex splicing, and multiple promoters. Genomics 90 (3):397-406.

Pyo, C. W., S. H. Lee, \& S. Y. Choi. 2008. Oxidative stress induces PKR-dependent apoptosis via IFN-gamma activation signaling in Jurkat $\mathrm{T}$ cells. Biochemical and biophysical research communications 377 (3):1001-6.

Rebbapragada, I., \& J. Lykke-Andersen. 2009. Execution of nonsense-mediated mRNA decay: what defines a substrate? Current opinion in cell biology 21 (3):394-402.

Richter, J. D. 2007. CPEB: a life in translation. Trends in biochemical sciences 32 (6):279-85.

Robertson, J., et al. 2003. A neurotoxic peripherin splice variant in a mouse model of ALS. The Journal of cell biology 160 (6):939-49.

Russo, A., et al. 2008. cis-acting sequences and trans-acting factors in the localization of mRNA for mitochondrial ribosomal proteins. Biochimica et biophysica acta 1779 (12):820-9.

Savas, J. N., et al. 2010. A role for huntington disease protein in dendritic RNA granules. The Journal of biological chemistry 285 (17):13142-53.

Savas, J. N., et al. 2008. Huntington's disease protein contributes to RNA-mediated gene silencing through association with Argonaute and $\mathrm{P}$ bodies. Proceedings of the National Academy of Sciences of the United States of America 105 (31):10820-5.

Schwab, C., et al. 2008. Colocalization of transactivation-responsive DNA-binding protein 43 and huntingtin in inclusions of Huntington disease. Journal of neuropathology and experimental neurology 67 (12):1159-65.

Sephton, C. F., et al. 2011. Identification of neuronal RNA targets of TDP-43-containing ribonucleoprotein complexes. The Journal of biological chemistry 286 (2):1204-15.

Seredenina, T., \& R. Luthi-Carter. 2011. What have we learned from gene expression profiles in Huntington's disease? Neurobiology of disease.

Shinchuk, L. M., et al. 2005. Poly-(L-alanine) expansions form core beta-sheets that nucleate amyloid assembly. Proteins 61 (3):579-89.

Siomi, H., \& M. C. Siomi. 2009. On the road to reading the RNA-interference code. Nature 457 (7228):396-404.

Sisodia, S. S. 1992. Beta-amyloid precursor protein cleavage by a membrane-bound protease. Proceedings of the National Academy of Sciences of the United States of America 89 (13):6075-9.

Sossin, W. S., \& L. DesGroseillers. 2006. Intracellular trafficking of RNA in neurons. Traffic 7 (12):1581-9.

Spriggs, K. A., M. Bushell, \& A. E. Willis. 2010. Translational regulation of gene expression during conditions of cell stress. Molecular cell 40 (2):228-37.

St Johnston, D., D. Beuchle, \& C. Nusslein-Volhard. 1991. Staufen, a gene required to localize maternal RNAs in the Drosophila egg. Cell 66 (1):51-63. 
Strong, M. J., et al. 2007. TDP43 is a human low molecular weight neurofilament (hNFL) mRNA-binding protein. Molecular and cellular neurosciences 35 (2):320-7.

Swanger, S. A., \& G. J. Bassell. 2011. Making and breaking synapses through local mRNA regulation. Current opinion in genetics $\mathcal{E}$ development 21 (4):414-21.

Tatom, J. B., et al. 2009. Mimicking aspects of frontotemporal lobar degeneration and Lou Gehrig's disease in rats via TDP-43 overexpression. Molecular therapy : the journal of the American Society of Gene Therapy 17 (4):607-13.

Tian, B., et al. 2005. A large-scale analysis of mRNA polyadenylation of human and mouse genes. Nucleic acids research 33 (1):201-12.

Trettel, F., et al. 2000. Dominant phenotypes produced by the HD mutation in STHdh(Q111) striatal cells. Human molecular genetics 9 (19):2799-809.

Van Dam, D., et al. 2005. Cognitive decline, neuromotor and behavioural disturbances in a mouse model for fragile-X-associated tremor/ataxia syndrome (FXTAS). Behavioural brain research 162 (2):233-9.

Vance, C., et al. 2009. Mutations in FUS, an RNA processing protein, cause familial amyotrophic lateral sclerosis type 6. Science 323 (5918):1208-11.

Venter, J. C., et al. 2001. The sequence of the human genome. Science 291 (5507):1304-51.

Villace, P., R. M. Marion, \& J. Ortin. 2004. The composition of Staufen-containing RNA granules from human cells indicates their role in the regulated transport and translation of messenger RNAs. Nucleic acids research 32 (8):2411-20.

Volkening, K., et al. 2009. Tar DNA binding protein of $43 \mathrm{kDa}$ (TDP-43), 14-3-3 proteins and copper/zinc superoxide dismutase (SOD1) interact to modulate NFL mRNA stability. Implications for altered RNA processing in amyotrophic lateral sclerosis (ALS). Brain research 1305:168-82.

Wahl, M. C., C. L. Will, \& R. Luhrmann. 2009. The spliceosome: design principles of a dynamic RNP machine. Cell 136 (4):701-18.

Wang, D. O., K. C. Martin, \& R. S. Zukin. 2010. Spatially restricting gene expression by local translation at synapses. Trends in neurosciences 33 (4):173-82.

Weigell-Weber, M., W. Schmid, \& R. Spiegel. 1996. Psychiatric symptoms and CAG expansion in Huntington's disease. American journal of medical genetics 67 (1):53-7.

Wellington, C. L., et al. 1998. Caspase cleavage of gene products associated with triplet expansion disorders generates truncated fragments containing the polyglutamine tract. The Journal of biological chemistry 273 (15):9158-67.

Willemsen, R., J. Levenga, \& B. Oostra. 2011. CGG repeat in the FMR1 gene: size matters. Clinical genetics 80 (3):214-25.

Xiao, S., S. Tjostheim, et al. 2008. An aggregate-inducing peripherin isoform generated through intron retention is upregulated in amyotrophic lateral sclerosis and associated with disease pathology. The Journal of neuroscience : the official journal of the Society for Neuroscience 28 (8):1833-40.

$\mathrm{Xu}$, Z., et al. 1993. Increased expression of neurofilament subunit NF-L produces morphological alterations that resemble the pathology of human motor neuron disease. Cell 73 (1):23-33.

Zhou, W., and W. Song. 2006. Leaky scanning and reinitiation regulate BACE1 gene expression. Molecular and cellular biology 26 (9):3353-64. 
$\mathrm{Zu}$, T., et al. 2011. Non-ATG-initiated translation directed by microsatellite expansions. Proceedings of the National Academy of Sciences of the United States of America 108 (1):260-5.

Zuccato, C., et al. 2001. Loss of huntingtin-mediated BDNF gene transcription in Huntington's disease. Science 293 (5529):493-8. 


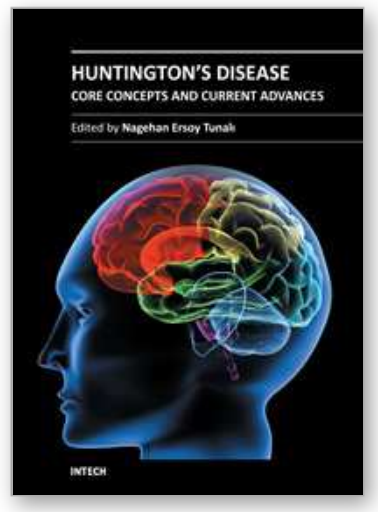

\author{
Huntington's Disease - Core Concepts and Current Advances \\ Edited by Dr Nagehan Ersoy Tunali
}

ISBN 978-953-307-953-0

Hard cover, 554 pages

Publisher InTech

Published online 15, February, 2012

Published in print edition February, 2012

Huntington's Disease is one of the well-studied neurodegenerative conditions, a quite devastating and currently incurable one. It is a brain disorder that causes certain types of neurons to become damaged, causing various parts of the brain to deteriorate and lose their function. This results in uncontrolled movements, loss of intellectual capabilities and behavioural disturbances. Since the identification of the causative mutation, there have been many significant developments in understanding the cellular and molecular perturbations. This book, "Huntington's Disease - Core Concepts and Current Advances", was prepared to serve as a source of up-to-date information on a wide range of issues involved in Huntington's Disease. It will help the clinicians, health care providers, researchers, graduate students and life science readers to increase their understanding of the clinical correlates, genetic aspects, neuropathological findings, cellular and molecular events and potential therapeutic interventions involved in HD. The book not only serves reviewed fundamental information on the disease but also presents original research in several disciplines, which collectively provide comprehensive description of the key issues in the area.

\title{
How to reference
}

In order to correctly reference this scholarly work, feel free to copy and paste the following:

Brady P. Culver and Naoko Tanese (2012). Role of Huntington's Disease Protein in Post-Transcriptional Gene Regulatory Pathways, Huntington's Disease - Core Concepts and Current Advances, Dr Nagehan Ersoy Tunali (Ed.), ISBN: 978-953-307-953-0, InTech, Available from: http://www.intechopen.com/books/huntington-sdisease-core-concepts-and-current-advances/role-of-huntington-s-disease-protein-in-post-transcriptionalgene-regulatory-pathways

\section{INTECH}

open science | open minds

\section{InTech Europe}

University Campus STeP Ri

Slavka Krautzeka 83/A

51000 Rijeka, Croatia

Phone: +385 (51) 770447

Fax: +385 (51) 686166

www.intechopen.com

\section{InTech China}

Unit 405, Office Block, Hotel Equatorial Shanghai

No.65, Yan An Road (West), Shanghai, 200040, China

中国上海市延安西路65号上海国际贵都大饭店办公楼 405 单元

Phone: +86-21-62489820

Fax: $+86-21-62489821$ 
(C) 2012 The Author(s). Licensee IntechOpen. This is an open access article distributed under the terms of the Creative Commons Attribution 3.0 License, which permits unrestricted use, distribution, and reproduction in any medium, provided the original work is properly cited. 